\title{
NONLINEAR DISSIPATIVE WAVE EQUATIONS WITH POTENTIAL
}

\author{
GROZDENA TODOROVA AND BORISLAV YORDANOV
}

\begin{abstract}
We study the long time behavior of solutions of the wave equation with absorbtion $|u|^{p-1} u$ and variable damping $V(x) u_{t}$, where $V(x) \sim V_{0}|x|^{-\alpha}$ for large $|x|, V_{0}>0$. For $\alpha \in[0,1)$ and $1<p<(n+2) /(n-2)$ we establish decay estimates of the energy, $L^{2}$ and $L^{p+1}$ norm of solutions. We find three different regimes of decay of solutions depending on the exponent of the absorbtion term. We show that $p_{1}(n, \alpha):=1+2\left(4-\alpha^{2}\right) /[(n-\alpha)(4-\alpha)]$ is a critical exponent in the following sense. For the supercritical region, namely $p_{1}(n, \alpha)<p<(n+2) /(n-2)$, the decay of solutions of the nonlinear equation coincides with the decay of the corresponding linear problem. For the subcritical region $1<p<p_{1}(n, \alpha)$ the decay is much faster. Moreover, the subcritical region $1<p<p_{1}(n, \alpha)$ is divided by two subregions with completely different decay rates by another critical exponent $p_{2}(n, \alpha):=1+2 \alpha /(n-\alpha)$. The decay rate of solutions becomes independent of $\alpha$ if $1<p<p_{2}(n, \alpha)$. Deriving the decay of solutions even for the linear problem with potential $V(x)$ is a delicate task and requires serious strengthening of the multiplier method. We also consider the decay problems for nonlinear damped equation with absorbtion under more general assumptions on the potential.
\end{abstract}

\section{INTRODUCTION}

In this paper we study the decay properties of solutions to the initial value problem

$$
\begin{aligned}
& u_{t t}-\Delta u+V(x) u_{t}+|u|^{p-1} u=0, \quad x \in \mathbf{R}^{n}, \quad t>0, \\
& u(0, x)=u_{0}(x), \quad u_{t}(0, x)=u_{1}(x),
\end{aligned}
$$

in dimensions $n \geq 3$, where $1<p<(n+2) /(n-2)$. The potential $V$ is a radial $C^{1}$-function of $x$ satisfying the inequalities

$$
V_{0}(1+|x|)^{-\alpha} \leq V(x) \leq V_{1}(1+|x|)^{-\alpha}, \quad \alpha \in[0,1),
$$

for constants $V_{0}, V_{1}>0$. Concerning the initial data, we assume that these are compactly supported and belong to the energy space:

$$
u_{0} \in H^{1}\left(\mathbf{R}^{n}\right), \quad u_{1} \in L^{2}\left(\mathbf{R}^{n}\right), \quad u_{0}(x) \text { and } u_{1}(x)=0 \text { for }|x|>R .
$$

Under these conditions, problem (1.1) admits a global solution $u$, such that

$$
u \in C\left((0, \infty), H^{1}\left(\mathbf{R}^{n}\right)\right), \quad u_{t} \in C\left((0, \infty), L^{2}\left(\mathbf{R}^{n}\right)\right) .
$$

Moreover, the finite propagation speed of (1.1) yields

$$
u(t, x)=0 \text { for }|x|>t+R,
$$

see Strauss [S3], Shatah and Struwe [S1S2].

\footnotetext{
${ }^{1}$ Research of the first author supported in part by NSF grant DMS-0245578

1991 Mathematics Subject Classification. Primary 35L05, 35L70; Secondary 37L15.

Key words and phrases. wave equation, weak dissipation, decay rates.
} 
Let us recall the definition of energy associated with a solution of (1.1):

$$
E(t)=\frac{1}{2} \int\left(u_{t}^{2}+|\nabla u|^{2}\right) d x+\frac{1}{p+1} \int|u|^{p+1} d x,
$$

where the integration takes place over $\mathbf{R}^{n}$. This quantity decays in time as a result of the conservation law

$$
E(t)=E(0)-\int_{0}^{t} \int V u_{s}^{2} d x d s .
$$

The decay rate, however, is not evident from such conservation laws.

We are also interested in the asymptotic behavior of the $L^{2}, L^{p+1}$ and energy norms of $u$ :

$$
\|u\|_{L^{2}}, \quad\|u\|_{L^{p+1}} \text { and }\left\|u_{t}\right\|_{L^{2}}+\|\nabla u\|_{L^{2}}
$$

respectively.

Hence the energy is a non-increasing function of $t$. It is a natural question whether the energy decays to zero as $t$ goes to infinity and if so, how fast it decays. Of course a lower bound on $V$ will be necessary, since the solutions are asymptotically free if $V$ decays very fast. It is known that the energy of $u$ approaches a non-zero constant as $t \rightarrow \infty$ if $V(x)=O\left(|x|^{-1-\delta}\right)$ with some $\delta>0$, see Mochizuki [M3].

There are many papers where the decay problems of solutions $u$ to the Cauchy problem for the damped wave equations with absorption (1.1) and potential $V(x)=$ 1 have been treated. Due to the diffusion phenomena the behavior of $u$ as $t \rightarrow \infty$ is expected to be the same as the corresponding heat equation

$$
u_{t}-\Delta u+|u|^{p-1} u=0, \quad x \in \mathbf{R}^{n}, \quad t>0 .
$$

Kawashima, Nakao and Ono in [KNO] consider the decay problem for large exponent $p$ of the absorption where the decay rate is expected to be as that of the linear heat equation. The authors of [KNO] prove this only for very large $p$, namely for

$$
p>1+\frac{4}{n} \text { and } 1 \leq n \leq 3,
$$

by using the energy method combined with $L^{p}-L^{q}$ estimates. They show that if

$$
1+\frac{4}{n}<p<\frac{n+2}{n-2} \text { for } n=3 \text { or } 1+\frac{4}{n}<p<\infty \quad(n=1,2)
$$

the solution $u(t, x)$ decays as

$$
\|u\|_{L^{2}}=O\left(t^{-\frac{n}{2}\left(\frac{1}{r}-\frac{1}{2}\right)}\right),
$$

when the data $\left(u_{0}, u_{1}\right) \in\left(H^{1} \cap L^{r}\left(\mathbf{R}^{n}\right) \times\left(L^{2} \cap L^{r}\left(\mathbf{R}^{n}\right)\right.\right.$ for $1 \leq r \leq 2$.

Let us mention here the paper [TY1] where the authors prove for the equation with a forcing nonlinearity

$$
u_{t t}-\Delta u+u_{t}=|u|^{p}, \quad x \in \mathbf{R}^{n}, \quad t>0
$$

in the supercritical case namely $p>1+\frac{2}{n}$ that any small data solutions are global and their decay rate is like the decay rate of the linear heat equation

$$
\|D u\|_{L^{2}}=O\left(t^{-\frac{n}{4}-\frac{1}{2}}\right)
$$

where $D=\left(\frac{\partial}{\partial t}, \nabla_{x}\right)$. 
Based on $[\mathrm{KNO}]$ paper Karch $[\mathrm{K}]$ shows that the asymptotic profile of the solution $u$ is the Gauss kernel. Again the above result was proved only for very large $p$ and small $n$ namely

$$
p>1+\frac{4}{n} \text { and } 1 \leq n \leq 3
$$

Only in dimension $n=1$ in a very recent paper [HKN] the authors succeeded to close the gap and prove that the asymptotic profile of $u$ for the supercritical case

$$
p>1+\frac{2}{n} \text { and } n=1
$$

is the Gauss kernel.

The decay rate of the solution in subcritical case, namely with small exponent

$$
1<p<1+\frac{2}{n}
$$

of the absorption term was expected to be the decay of the corresponding nonlinear heat equation (1.3). In a very recent paper [NZ] Nishihara and Zhao prove this for the exponentially decaying data as $x \rightarrow \infty$, by using the weighted energy method with convenient weights similar to the weights in [TY1]. They show that

$$
\left(\|u\|_{L^{p+1}},\|\nabla u\|_{L^{2}}\right)=O\left(t^{-\frac{1}{p-1}+\frac{n}{2(p+1)}}, t^{-\frac{1}{p-1}+\frac{n}{4}-\frac{1}{2}}\right),
$$

for $1<p<1+2 / n$, which coincides with the decay results of Escobedo and Kavian $[\mathrm{EK}]$ for the corresponding norms of the semi-linear heat equation.

In this paper we consider the decay problems of solutions for the nonlinear damped wave equation (1.1) with potential $V(x)$ in both cases supercritical and subcritical. Of course the critical number as well as the decay rates are quite different than the case of constant potential $V(x)=1$. Moreover we observe a new effect which does not show up in the case of constant potential. Unlike the decay problems for constant potential were there are two different decay rate regions, in the case of potential $V(x)$ we observe three different decay rate regions dependent on the sizes of the exponent of nonlinearity. The decay rate for the nonlinear problem is closely related with the decay rate of the linear damped wave equation in the region of large exponents of the nonlinearity. This problem is quite delicate and deserves separate consideration.

Therefore, before stating our main results we will consider the decay rate problems for the linear damped equation with potential $V(x)$

$$
u_{t t}-\Delta u+V(x) u_{t}=0, \quad x \in \mathbf{R}^{n}, \quad t>0,
$$

where $V(x)$ is a positive $C^{1}$-coefficient. There are many papers where the decay rate problems for the linear damped wave equation with a potential are treated. When the potential is a function of time $V(t)$, the Fourier analysis remains a powerful tool to handle the problem. A series of sharp $L^{p}-L^{q}$ estimates are derived in the papers of Wirth [W1], [W2], Reissig [Ra], Reissg and Wirth [RW], by using the Fourier transform method for potential $V(t)=a_{0}(1+t)^{k}$ and $k \in(-1,1)$.

When the potential is $V(x)$ in (1.4) the Fourier techniques become cumbersome. A simple alternative is the multiplier method. Below we discuss several results found by non-trivial applications of this method. Matsumura [M2] and Uesaka [U] have established decay estimates for the energy when the coefficient of the damping term has critical decay

$$
V(t, x) \geq V_{0}(1+t+|x|)^{-1},
$$


with $V_{0}>0$. (We omit additional technical conditions on $V$.) Their results can be stated as

$$
\int\left(u_{t}^{2}+|\nabla u|^{2}\right) d x \leq C_{0} t^{-\min \left\{2, V_{0}\right\}} .
$$

The basic multiplier is $\{w(t) u\}_{t}$ with a suitable weight $w(t)$. Remarkably this method can handle the critical decay but can not take advantage of any slower decay, such as

$$
V(x) \geq V_{0}(1+|x|)^{-\alpha}, \quad \alpha \in[0,1) .
$$

In fact estimate (1.5) does not remain sharp for stronger dissipations; notice that the decay rate of energy should be close to $t^{-n / 2-1}$ if $\alpha$ is close to 0 .

Multipliers depending on $t$ and $x$ yield sharper estimates for (1.1). The equation is studied in the exterior of star-shaped domains by Nakao [N1], Mochizuki and Nakazawa [MN], and Ikehata and Matsuyama [IM]. If $n \geq 2$ and

$$
V(x) \in L^{\infty}, \quad V(x) \geq V_{0}
$$

for large $x$, the result of [IM] is that the energy decays like $t^{-2}$. This decay is sharp only if $n=2$.

Another application of the multiplier method is the weighted estimate of Ikehata [I] for coefficients

$$
V(x)=V_{0}|x|^{-\alpha}, \quad \alpha \in[0,1) .
$$

Using an exponential multiplier, he has established the following:

$$
\int e^{2(2-\alpha)^{-2} V_{0} \frac{|x|^{2-\alpha}}{t}}\left(u_{t}^{2}+|\nabla u|^{2}\right) d x \leq C_{0},
$$

for $t \geq 1$. Hence $u$ decays very fast if $V_{0}>0$ and $|x|^{2-\alpha} / t$ is large. If this ratio is small, the result does not provide new information.

In general multiplier techniques yield weaker decay estimates than Fourier techniques whenever the latter can be applied to (1.1). The discrepancy may be explained with the equal weight of $u_{t}^{2}$ and $|\nabla u|^{2}$ in the standard multiplier method, while these terms are very different at large times. In fact the so-called diffusion phenomenon is observed for $V=1$, which means that $u \sim C_{0} w$ for a constant $C_{0}$ depending on the initial data and a solution $w$ of the diffusion equation

$$
w_{t}-\Delta w=0, \quad x \in \mathbf{R}^{n}, \quad t>0 .
$$

See the review by Narazaki [N2]. We can actually use a Gaussian

$$
w(t, x)=t^{-\frac{n}{2}} e^{-\frac{|x|^{2}}{4 t}} .
$$

Clearly the time derivative of the Gaussian is smaller then its spatial derivatives, i.e., $\left\|w_{t}\right\|_{L^{2}}$ is smaller than $\|\nabla w\|_{L^{2}}$.

This observation gives us an idea how to strengthen the multiplier method. If we can find an approximate solution $w$ of (1.1) and factor out the asymptotic profile $w$ we can work with $w^{-1} u$. The quotient $w^{-1} u$ will vary relatively slowly and will admit more precise estimates. This approach relies on three components.

1. Find an approximate solution of (1.1).

2. Derive a modified equation for $w^{-1} u$ and weighted identities.

3. Choose weights to derive the optimal decay rate for $u$.

Unexpectedly $w$ is allowed to be even an approximate solution of the inequality

$$
w_{t t}+V(x) w_{t}-\Delta w \geq-f(t, x) w,
$$


with a suitable function $f$ decaying sufficiently fast at infinity. In fact the approximate solution of the inequality (1.7) can be chosen as

$$
w(t, x)=t^{-m} e^{-\beta \frac{\phi(x)}{t}},
$$

with suitable parameters $m, \beta$. In the above $\phi$ is a positive solution of the Poisson equation

$$
\Delta \phi(x)=V(x), \quad x \in \mathbf{R}^{n} .
$$

The choice of weights and of the function $f$ in (1.7) is delicate. It turns out that the sharpest estimates for high exponents $p$ are obtained when $f=0$; low exponents $p$ require $f>0$.

The decay rate of $u$ will be expressed in terms of $w$ and, implicitly, in terms of the coefficient $V$. In fact we derive explicit rates in several cases. These decay estimates are sharp at the limit $\alpha \rightarrow 0$ in (1.2). The corresponding results for the linear damped equation with potential (1.4) are already obtained in [TY2].

To state the main decay estimates for the nonlinear equation (1.1) we need a special solution of the Poisson equation, see [TY2].

Proposition 1.1. Assume that $V(x)$ is radially symmetric and satisfies condition (1.2). Then equation (1.9) admits a solution $\phi \in C^{2}\left(\mathbf{R}^{n}\right)$, such that

$$
\begin{aligned}
& \phi_{0}(1+|x|)^{2-\alpha} \leq \phi(x) \leq \phi_{1}(1+|x|)^{2-\alpha}, \\
& m(V):=\liminf _{x \rightarrow \infty} \frac{V(x) \phi(x)}{|\nabla \phi(x)|^{2}}>0,
\end{aligned}
$$

where $\phi_{0}$ and $\phi_{1}$ are positive constants.

Let us consider an important class of admissible potentials $V(x)$.

Example 1.2. Assume that $V(x)>0$ is a radially symmetric $C^{1}$-function and

$$
V(x) \sim V_{2}|x|^{-\alpha}, \quad|x| \rightarrow \infty,
$$

with $V_{2}>0$; then condition (1.2) is trivially satisfied. It is shown in [TY2] that the Poisson equation (1.9) has a solution with the following behavior:

$$
\phi(x) \sim \frac{V_{2}}{(2-\alpha)(n-\alpha)}|x|^{2-\alpha}, \quad|\nabla \phi(x)| \sim \frac{V_{2}}{n-\alpha}|x|^{1-\alpha}, \quad|x| \rightarrow \infty .
$$

In this case the limit $m(V)$ is readily computed:

$$
m(V)=\frac{n-\alpha}{2-\alpha} .
$$

Now we are ready to state the first decay estimates for the solution of the nonlinear wave equation (1.1).

Theorem 1.3. Let $1<p<(n+2) /(n-2)$ and assume that $V$ satisfies (1.2). Then the solution of (1.1) satisfies

$$
\begin{aligned}
\int e^{(m(V)-\delta) \frac{\phi(x)}{t}} u^{2} d x & \leq c_{\delta} t^{\delta+\frac{\alpha}{2-\alpha}-m(V)}, \\
\int e^{(m(V)-\delta) \frac{\phi(x)}{t}}\left(u_{t}^{2}+|\nabla u|^{2}\right) d x & \leq c_{\delta} t^{\delta-1-m(V)} \\
\int e^{(m(V)-\delta) \frac{\phi(x)}{t}}|u|^{p+1} d x & \leq c_{\delta} t^{\delta-1-m(V)}
\end{aligned}
$$


where $\delta>0$ is an arbitrary small number and $t \geq 1$. The constant $c_{\delta}$ depends on $\delta$ and the initial data $u_{0}$ and $u_{1}$.

This decay rate is optimal only for large exponent $p$. The next result may be sharper when the exponent $p$ is close to 1 .

Theorem 1.4. Let $1<p<(n+2) /(n-2)$ and assume that $V$ satisfies (1.2). Then the solution of (1.1) satisfies

$$
\begin{aligned}
\int e^{(m(V)-\delta) \frac{\phi(x)}{t}} u^{2} d x & \leq c_{\delta} t^{\delta+\frac{\alpha}{2-\alpha}-\frac{2}{p-1}-\min \left\{\frac{\alpha}{2} \frac{p+1}{p-1}-\frac{n}{2}, 0\right\}}, \\
\int e^{(m(V)-\delta) \frac{\phi(x)}{t}}\left(u_{t}^{2}+|\nabla u|^{2}\right) d x & \leq c_{\delta} t^{\delta-\frac{p+1}{p-1}-\min \left\{\frac{\alpha}{2} \frac{p+1}{p-1}-\frac{n}{2}, 0\right\}}, \\
\int e^{(m(V)-\delta) \frac{\phi(x)}{t}}|u|^{p+1} d x & \leq c_{\delta} t^{\delta-\frac{p+1}{p-1}-\min \left\{\frac{\alpha}{2} \frac{p+1}{p-1}-\frac{n}{2}, 0\right\}},
\end{aligned}
$$

where $\delta>0$ is an arbitrary small number and $t \geq 1$. The constant $c_{\delta}$ depends on $\delta$ and on the initial data $u_{0}$ and $u_{1}$.

An important observation for both theorems is the following: the average decay rate of all norms under consideration in the region $\phi(x) \geq t^{1+\epsilon}, \epsilon>0$, is exponential. In fact we can add the three estimates in Theorem 1.3 or Theorem 1.4 to obtain the following.

Corollary 1.5. Let $1<p<(n+2) /(n-2)$ and assume that $V$ satisfies (1.2). Then the solution of (1.1) satisfies the estimate

$$
\int_{\phi(x) \geq t^{1+\epsilon}}\left(u^{2}+u_{t}^{2}+|\nabla u|^{2}+|u|^{p+1}\right) d x \leq c_{\delta} e^{-(m(V)-\delta) t^{\epsilon}} .
$$

where $c_{\delta}$ is a positive constant which depends on $\alpha, p$, and $n$.

Remark 1.6. Another interesting observation is that the decay of $\|u\|_{p+1}^{p+1}$ is the same as the decay of the energy $\left\|u_{t}\right\|_{2}^{2}+\|\nabla u\|_{2}^{2}$ for all solution $u$ of problem (1.1). This estimate is much better than the estimate that can be derived by the standard interpolation inequality, namely the Gagliardo-Nirenberg inequality, and the independent decay estimates of $\|u\|_{2}$ and $\|\nabla u\|_{2}$. All other norms $\|u\|_{s}$ with $2<s<\frac{n+2}{n-2}$ and $s \neq p+1$ will have a decay rate lower then the decay rate of the energy. The exact calculation of the decay of these norms may be done by using the decay of $\|u\|_{2}$ and $\|\nabla u\|_{2}$ and Gagliardo-Nirenberg inequality.

The potentials described in Example 1.2 can be analyzed further. Since in this case $m(V)=\frac{n-\alpha}{2-\alpha}$ we derive from Theorem 1.3 the following explicit estimates.

Corollary 1.7. Let $V$ be a potential described in Example 1.2. Assume that

$$
p_{1}(n, \alpha):=1+\frac{2\left(4-\alpha^{2}\right)}{(n-\alpha)(4-\alpha)} \leq p<\frac{n+2}{n-2} .
$$

Then the solution of (1.1) satisfies the following estimates:

$$
\begin{aligned}
\int e^{V_{0}(2-\alpha+\delta)^{-2} \frac{|x|^{2-\alpha}}{t}} u^{2} d x & \leq c_{\delta} t^{\delta-\frac{n-2 \alpha}{2-\alpha}} \\
\int e^{V_{0}(2-\alpha+\delta)^{-2} \frac{|x|^{2-\alpha}}{t}}\left(u_{t}^{2}+|\nabla u|^{2}\right) d x & \leq c_{\delta} t^{\delta-1-\frac{n-\alpha}{2-\alpha}} \\
\int e^{V_{0}(2-\alpha+\delta)^{-2} \frac{|x|^{2-\alpha}}{t}}|u|^{p+1} d x & \leq c_{\delta} t^{\delta-1-\frac{n-\alpha}{2-\alpha}}
\end{aligned}
$$


where $t \geq 1$. Here $\delta>0$ is an arbitrary small number and $c_{\delta}$ depends on $\delta$ and on the initial data $u_{0}$ and $u_{1}$.

Corollary 1.7 shows that for the supercritical region

$$
p_{1}(n, \alpha) \leq p<\frac{n+2}{n-2}
$$

the decay of the solution of nonlinear problem coincides with the decay of the solution of corresponding linear problem, see [TY2] for comparison.

Remark 1.8. Let us mention that when the potential $V(x)=1$ it can be shown that $\delta=0$ in the above decay estimates. In this case we get the optimal decay, that was derived by Matsumura by using the Fourier technique. Moreover, unlike Matsumura's method our approach can work in exterior domains as well.

For the subcritical region $1<p<p_{1}(n, \alpha)$, Theorem 1.4 yields much faster decay rates.

Corollary 1.9. Let $V$ be a potential described in Example 1.2. Assume that

$$
1<p<p_{1}(n, \alpha) .
$$

Then the solution of (1.1) satisfies the following estimates:

$$
\begin{aligned}
\int e^{V_{0}(2-\alpha+\delta)^{-2} \frac{|x|^{2-\alpha}}{t}} u^{2} d x & \leq c_{\delta} t^{\delta+\frac{\alpha}{2-\alpha}-\frac{2}{p-1}-\min \left\{\frac{\alpha}{2} \frac{p+1}{p-1}-\frac{n}{2}, 0\right\}}, \\
\int e^{V_{0}(2-\alpha+\delta)^{-2} \frac{|x|^{2-\alpha}}{t}}\left(u_{t}^{2}+|\nabla u|^{2}\right) d x & \leq c_{\delta} t^{\delta-\frac{p+1}{p-1}-\min \left\{\frac{\alpha}{2} \frac{p+1}{p-1}-\frac{n}{2}, 0\right\}}, \\
\int e^{V_{0}(2-\alpha+\delta)^{-2} \frac{|x|^{2-\alpha}}{t}}|u|^{p+1} d x & \leq c_{\delta} t^{\delta-\frac{p+1}{p-1}-\min \left\{\frac{\alpha}{2} \frac{p+1}{p-1}-\frac{n}{2}, 0\right\}},
\end{aligned}
$$

where $\delta>0$ is an arbirary small number and $t \geq 1$. Here $c_{\delta}$ depends on $\delta$ and on the data $u_{0}$ and $u_{1}$.

Remark 1.10. The subcritical region $1<p<p_{1}(n, \alpha)$ is further divided into two subregions with completely different decay rates as follows.

For exponent $p$ very close to 1 namely $1<p<1+\frac{2 \alpha}{n-\alpha}=: p_{2}(n, \alpha)$ the decay rates are

$$
\left(\|u\|_{L^{p+1}},\|\nabla u\|_{L^{2}}+\left\|u_{t}\right\|_{L^{2}}\right)=O\left(t^{-\frac{1}{p-1}+\delta}, t^{-\frac{p+1}{2(p-1)}+\delta}\right),
$$

where $\delta$ is a small number. In this region the decay rate is independent of $\alpha$.

For the second subcritical region, namely the region of medium exponents $p$ $p_{2}(n, \alpha)<p<p_{1}(n, \alpha)$, the decay rate is

$$
\left(\|u\|_{L^{p+1}},\|\nabla u\|_{L^{2}}+\left\|u_{t}\right\|_{L^{2}}\right)=O\left(t^{-\left(1+\frac{\alpha}{2}\right) \frac{1}{p-1}+\frac{n}{2(p+1)}+\delta}, t^{-\left(1+\frac{\alpha}{2}\right) \frac{p+1}{2(p-1)}+\frac{n}{4}+\delta}\right),
$$

where $\delta$ is an arbirtary small number.

Remark 1.11. Let us mention that in the case of constant potential in (1.1) $(\alpha=0)$ the second critical exponent $p_{2}(n, \alpha)=1+\frac{2 \alpha}{n-\alpha}$ is equal to 1 which explains why in the case of constant potential there are only two different regions with respect to the decay rate of the solution of (1.1). In the case of constant potential $(\alpha=0)$ the first critical exponent $p_{1}(n, \alpha)=1+\frac{2\left(4-\alpha^{2}\right)}{(n-\alpha)(4-\alpha)}$ becomes the Fujita critical exponent $1+\frac{2}{n}$. 
Remark 1.12. A byproduct of the above corollaries is that the gap between the supercritical and subcritical regions is closed for any $n$ if the potential $V(x)$ is constant $(\alpha=0)$.

The paper is organized as follows. In section 2 we present the main weighted energy identities. In section 3 we derive the modified equation for $w^{-1} u$. In section 4 we derive the main weighted energy estimates. In section 5 we choose the weights and in section 6 we prove the main Theorems.

\section{A Weighted Energy Identity}

We derive an identity for first-order perturbations of the semi-linear wave equation. Similar identities will hold for all second-order hyperbolic equations. Our nonlinear equation has the form

$$
v_{t t}-\Delta v+A v_{t}+B \cdot \nabla v+C v+h|v|^{p-1} v=0
$$

with coefficients $A, B=\left(B_{1}, B_{2}, \ldots, B_{n}\right), C$ and $h \geq 0$ in $C^{1}\left((0, \infty) \times \mathbf{R}^{n}\right)$. It is convenient to assume that

$$
v(t, x)=0 \text { if }|x|>t+R,
$$

for some $R>0$, and

$$
v \in C\left((0, \infty), H^{2}\left(\mathbf{R}^{n}\right)\right) \cap C^{1}\left((0, \infty), H^{1}\left(\mathbf{R}^{n}\right)\right) .
$$

These conditions are simple but not optimal; later we use a standard approximation argument to establish the result for data in the energy space. Another direction for generalization is to allow data decaying exponentially fast at infinity.

Given $P$ and $Q$ in $C^{2}\left((0, \infty) \times \mathbf{R}^{n}\right)$, we form the multiplier $P v_{t}+Q v$. Equation (2.1) shows that

$$
\left(v_{t t}-\Delta v+A v_{t}+B \cdot \nabla v+C v\right)\left(P v_{t}+Q v\right)+h|v|^{p-1} v\left(P v_{t}+Q v\right)=0 .
$$

We will rewrite the left side in divergence form and apply Gauss' theorem. The argument is split into 5 steps.

Step 1. We multiply (2.1) by $v_{t}$ and rearrange the terms as follows:

$$
\left(\frac{v_{t}^{2}+|\nabla v|^{2}}{2}\right)_{t}-\nabla \cdot\left(v_{t} \nabla v\right)+A v_{t}^{2}+v_{t} B \cdot \nabla v+C\left(\frac{v^{2}}{2}\right)_{t}+h\left(\frac{|v|^{p+1}}{p+1}\right)_{t}=0,
$$

where the dot product is defined by $x \cdot y=x_{1} y_{1}+\cdots x_{n} y_{n}$.

Step 2. We now multiply (2.1) by $v$ and again rearrange the terms:

$$
\left(v_{t} v\right)_{t}-\nabla \cdot(v \nabla v)-v_{t}^{2}+|\nabla v|^{2}+A\left(\frac{v^{2}}{2}\right)_{t}+B \cdot \nabla \frac{v^{2}}{2}+C v^{2}+h|v|^{p+1}=0 .
$$

Step 3. Let us multiply the identity from Step 1 by $P$ and integrate over $\mathbf{R}^{n}$.

$$
\begin{aligned}
0= & \frac{d}{d t} \int P \frac{v_{t}^{2}+|\nabla v|^{2}}{2} d x-\int P_{t} \frac{v_{t}^{2}+|\nabla v|^{2}}{2} d x+\int \nabla P \cdot v_{t} \nabla v d x \\
& +\int\left(P A v_{t}^{2}+v_{t} P B \cdot \nabla v\right) d x+\frac{d}{d t} \int P C \frac{v^{2}}{2} d x-\int(P C)_{t} \frac{v^{2}}{2} d x \\
& +\frac{d}{d t} \int P h \frac{|v|^{p+1}}{p+1} d x-\int(P h)_{t} \frac{|v|^{p+1}}{p+1} d x .
\end{aligned}
$$

Notice that boundary terms vanish since the support of $v(t, \cdot)$ is compact. 
Step 4. We also multiply the identity from Step 2 by $Q$ and integrate over $\mathbf{R}^{n}$.

$$
\begin{aligned}
0= & \frac{d}{d t} \int Q v_{t} v d x-\int Q_{t} v_{t} v d x+\int \nabla Q \cdot v \nabla v d x \\
& +\int\left(Q|\nabla v|^{2}-Q v_{t}^{2}\right) d x+\frac{d}{d t} \int Q A \frac{v^{2}}{2} d x-\int(Q A)_{t} \frac{v^{2}}{2} d x \\
& -\int \nabla \cdot(Q B) \frac{v^{2}}{2} d x+\int Q C v^{2} d x+\int Q h|v|^{p+1} d x .
\end{aligned}
$$

Using

$$
Q_{t} v_{t} v=\frac{1}{2}\left(Q_{t} v^{2}\right)_{t}-\frac{1}{2} Q_{t t} v^{2}, \quad \nabla Q \cdot v \nabla v=\frac{1}{2} \nabla \cdot\left(v^{2} \nabla Q\right)-\frac{1}{2} v^{2} \Delta Q,
$$

we can rewrite the above identity as

$$
\begin{aligned}
0= & \frac{d}{d t} \int\left(Q v_{t} v-\frac{1}{2} Q_{t} v^{2}\right) d x+\int \frac{1}{2} Q_{t t} v^{2} d x-\int \frac{1}{2} \Delta Q v^{2} d x \\
& +\int\left(Q|\nabla v|^{2}-Q v_{t}^{2}\right) d x+\frac{d}{d t} \int Q A \frac{v^{2}}{2} d x-\int(Q A)_{t} \frac{v^{2}}{2} d x \\
& -\int \nabla \cdot(Q B) \frac{v^{2}}{2} d x+\int Q C v^{2} d x+\int Q h|v|^{p+1} d x .
\end{aligned}
$$

Step 5 We add the final identities from Step 3 and Step 4 and combine similar terms. This establishes the following result.

Proposition 2.1. Let $v \in C\left((0, \infty), H^{2}\left(\mathbf{R}^{n}\right)\right) \cap C^{1}\left((0, \infty), H^{1}\left(\mathbf{R}^{n}\right)\right)$ be a solution of (2.1) with compact support (2.2) and $h \geq 0$. For any pair of $C^{2}$-functions $P$ and $Q$, we have the equality

$$
\frac{d}{d t} E\left(v_{t}, \nabla v, v\right)+F\left(v_{t}, \nabla v\right)+G(v)+H(v)=0,
$$

where

$$
\begin{aligned}
E\left(v_{t}, \nabla v, v\right)= & \frac{1}{2} \int\left[P\left(v_{t}^{2}+|\nabla v|^{2}\right)+2 Q v_{t} v+\left(C P-Q_{t}+A Q\right) v^{2}\right. \\
& +\frac{1}{p+1} \int P h|v|^{p+1} d x \\
F\left(v_{t}, \nabla v\right)= & \frac{1}{2} \int\left(-P_{t}+2 A P-2 Q\right) v_{t}^{2} d x \\
& +\int(\nabla P+B P) \cdot v_{t} \nabla v d x \\
& +\frac{1}{2} \int\left(-P_{t}+2 Q\right)|\nabla v|^{2} d x \\
G(v)= & \frac{1}{2} \int\left[Q_{t t}-\Delta Q-(A Q)_{t}-\nabla \cdot(Q B)+2 C Q-(C P)_{t}\right] v^{2} d x \\
H(v)= & \int\left(Q h-\frac{1}{p+1}(P h)_{t}\right)|v|^{p+1} d x .
\end{aligned}
$$


A simple consequence is that $E(t)$ is bounded if $F(t)+G(t)+H(t) \geq-f(t)$ with $f \in L^{1}$. Next we relax the regularity conditions on $v$ showing that the same result holds for all $v$ in the energy space.

Corollary 2.2. Let $v \in C\left((0, \infty), H^{1}\left(\mathbf{R}^{n}\right)\right) \cap C^{1}\left((0, \infty), L^{2}\left(\mathbf{R}^{n}\right)\right)$ be a solution of equation (2.1) with compact support (2.2) and $h \geq 0$. Define the functionals $F, G$, $H$, and $E$ as in Proposition 2.1.

If there exists a non-negative function $f \in L^{1}\left(\mathbf{R}_{+}\right)$, such that

$$
F\left(v_{t}, \nabla v\right)+G(v)+H(v) \geq-f(t), \quad t \geq t_{0},
$$

then the weighted energy of $v$ satisfies

$$
E\left(v_{t}, \nabla v, v\right) \leq E_{0}+\int_{t_{0}}^{t} f(s) d s, \quad t \geq t_{0},
$$

where $E_{0}=\left.E\left(v_{t}, \nabla v, v\right)\right|_{t=t_{0}}$.

Proof. There is nothing to prove if $v(0, x) \in H^{2}$ and $v_{t}(0, x) \in H^{1}$. If we have $v(0, x) \in H^{1}$ and $v_{t}(0, x) \in L^{2}$, we find compactly supported regular sequences $v_{0}^{(k)}(x) \rightarrow v(0, x)$ in $H^{1}, v_{1}^{(k)}(x) \rightarrow v_{t}(0, x)$ in $L^{2}$ and denote the corresponding solutions of $(2.1)$ by $v^{(k)}$. Notice that $E\left(v_{t}, \nabla v, v\right)$ is controlled by the energy norm:

$$
\begin{aligned}
& \left|E\left(v_{t}^{(k)}, \nabla v^{(k)}, v^{(k)}\right)-E\left(v_{t}^{(l)}, \nabla v^{(l)}, v^{(l)}\right)\right| \\
\leq \quad & c(T, M)\left(\left\|v_{t}^{(k)}-v_{t}^{(l)}\right\|_{L^{2}}^{2}+\left\|\nabla v^{(k)}-\nabla v^{(l)}\right\|_{L^{2}}^{2}\right)
\end{aligned}
$$

whenever $t \in[0, T]$ and $\left\|v_{t}^{(k)}\right\|_{L^{2}}+\left\|\nabla v^{(k)}\right\|_{L^{2}} \leq M, \quad\left\|v_{t}^{(l)}\right\|_{L^{2}}+\left\|\nabla v^{(l)}\right\|_{L^{2}} \leq M$. The latter conditions hold with

$$
M=c(T)\left(\left\|v_{t}(0, \cdot)\right\|_{L^{2}}+\|\nabla v(0, \cdot)\|_{L^{2}}+\|v(0, \cdot)\|_{L^{p+1}}^{(p+1) / 2}\right)
$$

in fact $h \geq 0$ implies the following estimate for all $j$ and $t \in[0, T]$ :

$$
\left\|v_{t}^{(j)}\right\|_{L^{2}}+\left\|\nabla v^{(j)}\right\|_{L^{2}} \leq c(T)\left(\left\|v_{1}^{(j)}\right\|_{L^{2}}+\left\|\nabla v_{0}^{(j)}\right\|_{L^{2}}+\left\|v_{0}^{(j)}\right\|_{L^{p+1}}^{(p+1) / 2}\right) .
$$

The energy norm of solutions to problem (1.1) is a locally Lipschitz function of the initial data if $p \in[1,(n+2) /(n-2))$, see [S1S2] or [S3]. Hence

$$
\begin{aligned}
& \left|E\left(v_{t}^{(n)}, \nabla v^{(n)}, v^{(n)}\right)-E\left(v_{t}^{(k)}, \nabla v^{(k)}, v^{(k)}\right)\right| \\
\leq & c(T, M)\left(\left\|v_{1}^{(n)}-v_{1}^{(k)}\right\|_{L^{2}}^{2}+\left\|\nabla v_{0}^{(n)}-\nabla v_{0}^{(k)}\right\|_{L^{2}}^{2}\right),
\end{aligned}
$$

which yields

$$
\begin{aligned}
E\left(v_{t}, \nabla v, v\right) & =\lim _{n \rightarrow \infty} E\left(v_{t}^{(n)}, \nabla v^{(n)}, v^{(n)}\right) \\
& \leq\left.\lim _{n \rightarrow \infty} E\left(v_{t}^{(n)}, \nabla v^{(n)}, v^{(n)}\right)\right|_{t=t_{0}}+\int_{t_{0}}^{t} f(s) d s \\
& =E_{0}+\int_{t_{0}}^{t} f(s) d s .
\end{aligned}
$$




\section{The Modified Equation}

We will apply the estimate in Corollary 2.2 to $v=w^{-1} u$, where $u$ is a solution of (1.1) and $w>0$ is a smooth solution of the differential inequality

$$
w_{t t}-\Delta w+V(x) w_{t} \geq C^{-} w
$$

with a suitable $C^{-}$decaying sufficiently fast at infinity. The idea is to work with a new function $v$ which is asymptotically much simpler than $u$, see [TY2]. The sharpest estimates for high exponents $p$ are obtained when $C^{-}=0$; low exponents $p$ require certain $C^{-}>0$, see Section 5 .

To derive the modified equation for $v$, we substitute $u=v w$ into equation (1.1). This yields a first-order perturbation of the semi-linear wave equation:

$$
v_{t t}-\Delta v+A v_{t}+B \cdot \nabla v+C v+h|v|^{p-1} v=0,
$$

where the coefficients are given by

$$
\begin{aligned}
& A=V+2 w^{-1} w_{t}, \quad B=-2 w^{-1} \nabla w, \\
& C=w^{-1}\left(w_{t t}-\Delta w+V w_{t}\right), \quad h=w^{p-1} .
\end{aligned}
$$

In our application of Corollary 2.2 the multiplier $P v_{t}+Q v$ satisfies

$$
Q=w .
$$

The first coefficient $P$ and approximate solution $w$ will be determined later. Here we only give the simplified estimates resulting from $Q=w$.

Proposition 3.1. Let $u$ be a solution of (1.1) with compactly supported data

$$
u_{0} \in H^{1}, \quad u_{1} \in L^{2}
$$

and define $v=w^{-1} u$, where $w>0$ is a $C^{2}$-function. Assume that $Q$ is given at (3.4) and $C$ is given at (3.3). Then

$$
\begin{aligned}
E\left(v_{t}, \nabla v, v\right)= & \frac{1}{2} \int\left[P\left(v_{t}^{2}+|\nabla v|^{2}\right)+2 w v_{t} v+\left(C P+w_{t}+V w\right) v^{2}\right] d x \\
& +\frac{1}{p+1} \int P w^{p-1}|v|^{p+1} d x, \\
F\left(v_{t}, \nabla v\right)= & \frac{1}{2} \int\left(-P_{t}+2 V P+4 P(\ln w)_{t}-2 w\right) v_{t}^{2} d x \\
& +\int(\nabla P-2 P \nabla \ln w) \cdot v_{t} \nabla v d x \\
& +\frac{1}{2} \int\left(-P_{t}+2 w\right)|\nabla v|^{2} d x, \\
G(v)= & \frac{1}{2} \int\left[C w-(C P)_{t}\right] v^{2} d x, \\
H(v)= & \int\left(w^{p}-\frac{1}{p+1}\left(P w^{p-1}\right)_{t}\right)|v|^{p+1} d x .
\end{aligned}
$$

(We used $Q=w$ to simplify $E$ and $G$; we just replaced $Q$ by $w$ in $F$ and $H$.) 
Moreover, the condition $F\left(v_{t}, \nabla v\right)+G(v)+H(v) \geq-f(t)$ with a non-negative function $f \in L^{1}\left(\mathbf{R}_{+}\right)$implies that the weighted energy of $v$ satisfies

$$
E\left(v_{t}, \nabla v, v\right) \leq E_{0}+\int_{t_{0}}^{t} f(s) d s, \quad t \geq t_{0}
$$

where $E_{0}=\left.E\left(v_{t}, \nabla v, v\right)\right|_{t=t_{0}}$.

Proof. We apply Corollary 2.2 to equation (3.2) with $A, B, C$, and $h$ defined at (3.3). Recall that the functionals $F, G, H$, and $E$ are introduced in Proposition 2.1.

\section{Weighted Energy Estimates}

In this section we show that Proposition 3.1 applies to all positive weights $P$ and $w$ satisfying eight differential inequalities. Hence the $E\left(v_{t}, \nabla v, v\right)$ is a bounded function of time for such weights. Another problem is to show that the weighted energy is positive definite, so it can be used for decay estimates of $v$ and the original solution $u=w v$.

Proposition 4.1. Assume that $P>0$ and $w>0$ are $C^{1}$-functions, such that

(ii) $\quad-P_{t}+w \geq 0$,

(iii) $\quad\left(-P_{t}+2 w\right)\left(-P_{t}+2 V P+4 P(\ln w)_{t}-2 w\right) \geq(\nabla P-2 P \nabla \ln w)^{2}$,

(iv) $\quad(p+1) w^{p}-\left(P w^{p-1}\right)_{t} \geq w^{p}$.

Let $u$ be a solution of (1.1) in the energy space and define $v=w^{-1} u$. Then

$$
F\left(v_{t}, \nabla v\right)+G(v)+H(v) \geq-c \int w^{-1}\left|C^{-}\right|^{\frac{p+1}{p-1}} d x
$$

see the definitions of these functionals in Proposition 3.1. Thus the weighted energy $E\left(v_{t}, \nabla v, v\right)$ satisfies

$$
\begin{aligned}
E_{0}+c_{0} \geq & \frac{1}{2} \int\left[P\left(v_{t}^{2}+|\nabla v|^{2}\right)+2 w v_{t} v+\left(C P+w_{t}+V w\right) v^{2}\right] d x \\
& +\frac{1}{p+1} \int P w^{p-1}|v|^{p+1} d x
\end{aligned}
$$

for all $t \geq t_{0}$, where

$$
E_{0}=\left.E\left(v_{t}, \nabla v, v\right)\right|_{t=t_{0}}, \quad c_{0}=c \int_{t_{0}}^{\infty} \int w^{-1} \mid C^{-\mid \frac{p+1}{p-1}} d x d t .
$$

Proof. It follows from conditions (ii) and (iii) that $F\left(v_{t}, \nabla v\right) \geq 0$ as a nonnegative quadratic form of $v_{t}$ and $\nabla v$.

To deduce a lower bound on $G(v)$, we use condition $(i)$ and Young's inequality with exponents $(p+1) / 2$ and $(p+1) /(p-1)$ :

$$
\begin{aligned}
G(v) & =\frac{1}{2} \int\left[C w-(C P)_{t}\right] v^{2} d x \\
& \geq \frac{1}{2} \int C^{-} w v^{2} d x \\
& \geq-\int w^{p}|v|^{p+1} d x-c \int w^{-1} \mid C^{-\mid \frac{p+1}{p-1}} d x d t
\end{aligned}
$$


Condition (iv) yields

$$
H(v) \geq \int w^{p}|v|^{p+1} d x
$$

so we have

$$
G(v)+H(v) \geq-c \int w^{-1}\left|C^{-}\right|^{\frac{p+1}{p-1}} d x d t .
$$

We complete the proof we just apply Proposition 3.1.

It is important to have a positive definite weighted energy $E\left(v_{t}, \nabla v, v\right)$. Thus we need a suitable upper bound on the indefinite term $2 w v v_{t}$ under conditions $(i)-(i v)$. We will essentially rely on assumption (1.2) concerning the decay rate of $V$. Unfortunately there is an additional condition $(v)$ on $P$ and $w$ to guarantee that $C P v^{2}$ is dominated by the positive nonlinearity $P w^{p-1}|v|^{p+1}$.

Proposition 4.2. Assume that conditions (i)-(iv) in Proposition 4.1 hold for $P$ and $w$ and condition (1.2) holds for $V$. If

$$
\text { (v) } \quad C \geq C^{-} \text {and } \sup _{t \geq t_{0}} \int P w^{-2} \mid C^{-\frac{p+1}{p-1}} d x<\infty
$$

then

$$
\int w v^{2} d x \leq G_{0}+c\left(E_{0}+1\right) t^{\alpha}
$$

for $t \geq t_{0}$, where $G_{0}=\left.\int w v^{2} d x\right|_{t=t_{0}}$.

Proof. Notice that if we drop the derivatives in $E\left(v_{t}, \nabla v, v\right) \leq E_{0}+c_{0}$ from Proposition 4.1, the result is

$$
\begin{aligned}
& \frac{d}{d t} \int w v^{2} d x+\int V w v^{2} d x \\
\leq & 2\left(E_{0}+c_{0}\right)+\int\left|C^{-}\right| P v^{2} d x-\frac{2}{p+1} \int P w^{p-1}|v|^{p+1} d x .
\end{aligned}
$$

We use Young's inequality

$$
\left|C^{-}\right| P v^{2} \leq \frac{1}{p+1} P w^{p-1}|v|^{p+1}+c P w^{-2}\left|C^{-}\right|^{\frac{p+1}{p-1}}
$$

and introduce

$$
b_{0}=c \sup _{t \geq t_{0}} \int P w^{-2}\left|C^{-}\right|^{\frac{p+1}{p-1}} d x .
$$

Thus we obtain

$$
\frac{d}{d t} \int w v^{2} d x+\int V w v^{2} \leq 2\left(E_{0}+c_{0}\right)+b_{0}, \quad t \geq t_{0} .
$$

We can now derive an estimate of the weighted $L^{2}$-norm of $v$. From $|x| \leq t+R$ and condition (1.2) on $V$,

$$
\frac{d}{d t} \int w v^{2} d x+\frac{V_{0}}{(1+t+R)^{\alpha}} \int w v^{2} d x \leq c\left(E_{0}+1\right) .
$$

This inequality is equivalent to

$$
\frac{d}{d t}\left(e^{\frac{V_{0}}{1-\alpha}(1+t+R)^{1-\alpha}} \int w v^{2} d x\right) \leq c\left(E_{0}+1\right) e^{\frac{V_{0}}{1-\alpha}(1+t+R)^{1-\alpha}} .
$$


To complete the proof we integrate on $\left[t_{0}, t\right]$ and apply the following estimate:

$$
\int_{t_{0}}^{t} e^{c \tau^{1-\alpha}} d \tau=\frac{1}{1-\alpha} \int_{t_{0}^{1-\alpha}}^{t^{1-\alpha}} e^{c z} z^{\frac{\alpha}{1-\alpha}} d z \leq \frac{t^{\alpha} e^{c t^{1-\alpha}}}{c(1-\alpha)} .
$$

Combining last two results yields a weighted estimate of $v_{t}$ and $\nabla v$. Recall the inequality of Proposition 4.1:

$$
\begin{aligned}
2\left(E_{0}+c_{0}\right) \geq & \int\left[P\left(v_{t}^{2}+|\nabla v|^{2}\right)+2 w v_{t} v+\left(C P+w_{t}+V w\right) v^{2}\right] d x \\
& +\frac{2}{p+1} \int P w^{p-1}|v|^{p+1} d x .
\end{aligned}
$$

This result and $\left|2 v_{t} v\right| \leq \epsilon t^{\alpha} w v_{t}^{2}+\epsilon^{-1} t^{-\alpha} w v^{2}$, where $\epsilon \in(0,1)$, imply

$$
\begin{aligned}
& \int\left(P-\epsilon t^{\alpha} w\right)\left(v_{t}^{2}+|\nabla v|^{2}\right) d x+\int V w v^{2} d x \\
\leq & 2\left(E_{0}+c_{0}\right)+\int\left(\epsilon^{-1} t^{-\alpha} w-w_{t}\right) v^{2} d x \\
& +\int\left|C^{-}\right| P v^{2} d x-\frac{2}{p+1} \int P w^{p-1}|v|^{p+1} d x .
\end{aligned}
$$

Applying the argument of Proposition 4.2, we have that

$$
\begin{aligned}
& \int\left(P-\epsilon t^{\alpha} w\right)\left(v_{t}^{2}+|\nabla v|^{2}\right) d x+\int V w v^{2} d x+\frac{1}{p+1} \int P w^{p-1}|v|^{p+1} d x \\
\leq & 2\left(E_{0}+c_{0}\right)+b_{0}+\int\left(\epsilon^{-1} t^{-\alpha} w-w_{t}\right) v^{2} d x .
\end{aligned}
$$

The left side is positive and the right side is bounded by a constant if the weights $P$ and $w$ meet two additional conditions.

Proposition 4.3. Assume that $V$ satisfies (1.2). If the weights $P$ and $w$ satisfy conditions (i)-(iv) in Proposition 4.1, condition (v) in Proposition 4.2, and

$$
\begin{array}{cl}
(v i) & w \leq c_{1} t^{-\alpha} P \\
(\text { vii }) & \left|w_{t}\right| \leq c_{1} t^{-\alpha} w
\end{array}
$$

we have

$$
\begin{aligned}
\int P\left(v_{t}^{2}+|\nabla v|^{2}\right) d x & \leq c\left(G_{0}+E_{0}+1\right), \\
\int V w v^{2} d x & \leq c\left(G_{0}+E_{0}+1\right), \\
\int P w^{p-1}|v|^{p+1} d x & \leq c\left(G_{0}+E_{0}+1\right),
\end{aligned}
$$

for all $t \geq t_{0}$.

Proof. Estimate (4.1) and Proposition 4.2 show that $P-\epsilon t^{\alpha} w \geq P / 2$ and

$$
\int\left(\epsilon^{-1} t^{-\alpha} w-w_{t}\right) v^{2} d x \leq c \int w v^{2} d x
$$

are sufficient to establish the claim. These hold if we choose $\epsilon=\left(2 c_{1}\right)^{-1}$ with $c_{1}$ given in condition $(v i)$ and use condition (vii), respectively. 
Let us restate Proposition 4.2 and Proposition 4.3 in terms of $u$. Using $v=w^{-1} u$ we readily obtain

$$
\begin{aligned}
\int w^{-1} u^{2} d x & \leq G_{0}+c\left(E_{0}+1\right) t^{\alpha}, \\
\int V w^{-1} u^{2} d x & \leq c\left(G_{0}+E_{0}+1\right), \\
\int P w^{-2}|u|^{p+1} d x & \leq c\left(G_{0}+E_{0}+1\right) .
\end{aligned}
$$

However, we need a final assumption on $P$ and $w$ to deduce such estimates for $u_{t}$ and $\nabla u$. From $v=w^{-1} u$ we find that

$$
v_{t}^{2}=\left(-w^{-2} w_{t} u+w^{-1} u_{t}\right)^{2} \geq \frac{1}{2} w^{-2} u_{t}^{2}-3 w^{-4} w_{t}^{2} u^{2}
$$

and

$$
|\nabla v|^{2}=\left(-w^{-2} \nabla w u+w^{-1} \nabla u\right)^{2} \geq \frac{1}{2} w^{-2}|\nabla u|^{2}-3 w^{-4}|\nabla w|^{2} u^{2} .
$$

Proposition 4.3 and these inequalities imply

$$
\frac{1}{2} \int P w^{-2}\left(u_{t}^{2}+|\nabla u|^{2}\right) d x \leq c\left(G_{0}+E_{0}+1\right)+3 \int P w^{-4}\left(w_{t}^{2}+|\nabla w|^{2}\right) u^{2} d x .
$$

If the weights satisfy $P w^{-4}\left(w_{t}^{2}+|\nabla w|^{2}\right) \leq c_{2} V(x) w^{-1}$, we apply Proposition 4.2 to the right side. The final estimates are given below.

Proposition 4.4. Assume that $V$ satisfies (1.2) and $P$ and $w$ satisfy (i)-(vii) and

$$
P w^{-3}\left(w_{t}^{2}+|\nabla w|^{2}\right) \leq c_{2} V(x),
$$

where $c_{2}$ is a constant. Then the solution $u$ of (1.1) satisfies

$$
\begin{aligned}
\int V w^{-1} u^{2} d x & \leq c\left(G_{0}+E_{0}+1\right), \\
\int P w^{-2}\left(u_{t}^{2}+|\nabla u|^{2}\right) d x & \leq c\left(G_{0}+E_{0}+1\right), \\
\int P w^{-2}|u|^{p+1} d x & \leq c\left(G_{0}+E_{0}+1\right),
\end{aligned}
$$

for $t \geq t_{0}$. Here $c$ depends only on the equation and weights.

The above result yields non-trivial estimates if there exist weights with properties $(i)-(v i i i)$ which decay sufficiently fast as $t$ and $|x|$ go to infinity. Such weights $P$ and $w$ are already constructed for the linear equation in [TY2]. Here we find a new pair of weights adapter to the nonlinear term in problem (1.1). Both weights are defined in terms of certain positive solutions to the Laplace equation in $\mathbf{R}^{n}$. We provide more details in the next section.

\section{Existence of Good Weights}

We rely on results from [TY2] and Proposition 1.1 for the existence of weights satisfying all conditions $(i)-($ viii $)$. Given parameters $S_{0}>0$ and $\beta>0$, we introduce

$$
S(x)=\beta \phi(x)+S_{0}, \quad x \in \mathbf{R}^{n},
$$


where $\phi(x)$ is the function constructed in Proposition 1.1, and define the family of weights

$$
w(t, x)=t^{-m} e^{-\frac{S(x)}{t}}, \quad P(t, x)=\frac{3}{4}\left(\frac{6}{t}+\frac{S(x)}{t^{2}}\right)^{-1} w(t, x),
$$

where $m>0$ is an additional parameter. The two numbers $\beta$ and $m$ will depend on $n, p$ and $V$. Constants like $S_{0}, \frac{3}{4}$ and 6 are introduced for technical reasons; $S_{0}$ is a large number depending on $V$ and $n$ (see [TY2]). It is important to mention that $m$ determines the implicit decay rates as $t \rightarrow \infty$ in Proposition 4.4. Similarly, $S$ determines the decay of these norms restricted to the region $\left\{x: S(x) \geq t^{1+\epsilon}\right\}$, $\epsilon>0$.

The following result is established in [TY2].

Proposition 5.1. Let $w$ and $P$ be defined in (5.1). Then conditions (ii), (iii), and (vi)-(viii) on $w$ and $P$ hold for sufficiently large $t \geq t_{0}$.

The proof is straightforward so it will not be repeated here. Instead we verify the remaining conditions $(i),(i v)$, and $(v)$. Actually the condition $(i v)$ holds for all positive $m$ and $\beta$ in (5.1).

Proposition 5.2. Let $w$ and $P$ be defined in (5.1). Then condition (iv) holds for sufficiently large $t \geq t_{0}$.

Proof. Condition $(i v)$ is $(p+1) w^{p}-\left(P w^{p-1}\right)_{t} \geq w^{p}$ or $p w^{p}-\left(P w^{p-1}\right)_{t} \geq 0$. To show this we use condition (ii) and two simple computations:

$$
-P_{t}+w \geq 0, \quad \frac{w_{t}}{w}=-\frac{m}{t}+\frac{S(x)}{t^{2}}, \quad \frac{P}{w}=\frac{3}{4}\left(\frac{6}{t}+\frac{S(x)}{t^{2}}\right)^{-1} .
$$

Hence

$$
-\frac{P_{t}}{w} \geq-1, \quad \frac{P}{w} \frac{w_{t}}{w} \leq \frac{3}{4} .
$$

We now obtain

$$
\begin{aligned}
p w^{p}-\left(P w^{p-1}\right)_{t} & =p w^{p}-P_{t} w^{p-1}-P(p-1) w^{p-2} w_{t} \\
& =w^{p}\left(p-\frac{P_{t}}{w}-(p-1) \frac{P}{w} \frac{w_{t}}{w}\right) \\
& \geq w^{p}\left(p-1-\frac{3}{4}(p-1)\right) \geq 0 .
\end{aligned}
$$

The final two conditions $(i)$ and $(v)$ are related to the asymptotic behavior of $w$ as a solution to (3.1). We choose $C^{-}$so that $m$ is maximized. It turns out that the cases of low and high exponents $p$ are very different.

Let us compute the first and second order derivatives of $w$ :

$$
\begin{aligned}
& w_{t}=\left(-\frac{m}{t}+\frac{S(x)}{t^{2}}\right) w, \quad w_{t t}=\left(-\frac{m}{t}+\frac{S(x)}{t^{2}}\right)^{2} w+\left(\frac{m}{t^{2}}-\frac{2 S(x)}{t^{3}}\right) w \\
& \nabla w=-\frac{\nabla S(x)}{t} w, \quad \Delta w=\left(-\frac{\Delta S(x)}{t}+\frac{|\nabla S(x)|^{2}}{t^{2}}\right) w .
\end{aligned}
$$


Thus the coefficient $C$ in (3.3) is expressed as

$$
\begin{aligned}
C= & \frac{\Delta S-m V}{t}+\frac{V S-|\nabla S|^{2}}{t^{2}} \\
& +\left(-\frac{m}{t}+\frac{S(x)}{t^{2}}\right)^{2}+\frac{m}{t^{2}}-\frac{2 S(x)}{t^{3}} .
\end{aligned}
$$

We can now choose $m$ and $\beta$ so that conditions $(i)$ and $(v)$ are satisfied. Our first choice of parameters $m$ and $\beta$ will be optimal for supercritical exponents $p$. This choice is the following. Recall the definition of $m(V)$ in Proposition 1.1 and let $\delta>0$ be an arbitrary small number. We set

$$
m=m(V)-2 \delta, \quad \beta=m(V)-\delta .
$$

Proposition 5.3. Let $w$ and $P$ be defined in (5.1) and $m$ and $\beta$ be defined in (5.4). Then conditions $(i)$ and $(v)$ hold for sufficiently large $t \geq t_{0}$.

Proof. Assume that we have shown $C \geq 0$ and $C_{t} \leq 0$ for large $t \geq t_{0}$. Then we can take $C^{-}=0$. Thus the integrals in conditions $(i)$ and $(v)$ are trivially bounded. Moreover, from these assumptions and condition $(i i)$ we find

$$
C w-(C P)_{t}=C\left(w-P_{t}\right)-C_{t} P \geq 0 \text {. }
$$

Thus the inequality in condition $(i)$ also holds.

It remains to check $C \geq 0$ and $C_{t} \leq 0$. The crucial lower bounds are

$$
\Delta S(x) \geq(m+\delta) V(x), \quad(1-k \delta) V(x) S(x) \geq|\nabla S(x)|^{2} \text { for large }|x|,
$$

with $k=(1+m(V))^{-1}$; the latter bound follows from Proposition 1.1 and our definition of $S(x)$. From equality (5.3) we obtain

$$
\begin{aligned}
C & \geq \frac{\delta V(x)}{t}+\frac{k \delta V(x) S(x)-c}{t^{2}}-\frac{2 S(x)}{t^{3}} \\
& =\frac{\delta V(x)-c t^{-1}}{t}+\frac{S(x)\left(k \delta V(x)-2 t^{-1}\right)}{t^{2}}
\end{aligned}
$$

where $c$ is a constant depending on $V$. Notice that we can consider only $|x| \leq t+R$ in these estimates. Using $V(x) \geq V_{0}(1+|x|)^{-\alpha}$, with $\alpha \in[0,1)$, we see that $C>0$ for sufficiently large time $t$. The proof of $C_{t}<0$ is similar.

Our second choice of parameters $m$ and $\beta$ is optimal only for subcritical exponents $p$. We again fix a small $\delta>0$ and set

$$
\begin{aligned}
& m=\frac{2}{p-1}+\min \left\{\frac{\alpha}{2} \frac{p+1}{p-1}-\frac{n}{2}, 0\right\}-\delta, \\
& \beta=m(V)-\delta .
\end{aligned}
$$

Proposition 5.4. Let $w$ and $P$ be defined in (5.1) and $m$ and $\beta$ be defined in (5.5). Then conditions $(i)$ and $(v)$ hold for sufficiently large $t \geq t_{0}$.

Proof. We need estimates of $C$ and $C_{t}$ from below and above, respectively. 
Lemma 5.5. There exist positive constants $k_{i}, i=1,2,3,4$, such that

$$
\begin{aligned}
C(t, x) & \geq-k_{1} t^{-1}(1+|x|)^{-\alpha}+k_{2} t^{-2}(1+|x|)^{2-2 \alpha}, \\
t C_{t}(t, x) & \leq k_{3} t^{-1}(1+|x|)^{-\alpha}-k_{4} t^{-2}(1+|x|)^{2-2 \alpha} .
\end{aligned}
$$

Let us postpone the proof and apply the first estimate. We see that the lower bound of $C$ in condition $(v)$ can be chosen as

$$
C^{-}(t, x)= \begin{cases}-k_{1} t^{-1}(1+|x|)^{-\alpha}, & \text { if } 1+|x| \leq k t^{1 / 2} \\ 0, & \text { if } 1+|x|>k t^{1 / 2}\end{cases}
$$

where $k=\sqrt{\frac{k_{1}}{k_{2}}}$.

To verify the integral condition in $(v)$, we use the estimates $w^{-1}(t, x) P(t, x) \leq c t$ and $w(t, x) \geq c t^{m}$ on the support of $C^{-}$. The condition to check becomes

$$
\int P w^{-2}\left|C^{-}\right|^{\frac{p+1}{p-1}} d x \leq c t^{m+1-\frac{p+1}{p-1}} \int_{|x| \leq k t^{1 / 2}}(1+|x|)^{-\alpha \frac{p+1}{p-1}} d x .
$$

Notice that for every $\delta>0$ we have

$$
\int_{|x| \leq k t^{1 / 2}}(1+|x|)^{-\alpha \frac{p+1}{p-1}} d x \leq\left\{\begin{array}{l}
c_{\delta} t^{\left.\frac{n}{2}-\frac{\alpha}{2}\right) \frac{p+1}{p-1}+\delta}, \text { if } \alpha \frac{p+1}{p-1} \leq n \\
c, \text { if } \alpha \frac{p+1}{p-1}>n
\end{array}\right.
$$

Thus

$$
\int P w^{-2}\left|C^{-}\right|^{\frac{p+1}{p-1}} d x \leq\left\{\begin{array}{l}
c_{\delta} t^{m+1+\frac{n}{2}-\left(1+\frac{\alpha}{2}\right) \frac{p+1}{p-1}+\delta}, \text { if } \alpha \frac{p+1}{p-1} \leq n, \\
c t^{m+1-\frac{p+1}{p-1}}, \text { if } \alpha \frac{p+1}{p-1}>n .
\end{array}\right.
$$

Clearly the right sides are bounded functions of $t \geq t_{0}$ if $m$ is defined as (5.5).

The condition for integrability $(i)$ leads to similar estimates; we can show that

$$
\int_{t_{0}}^{\infty} \int w^{-1}\left|C^{-}\right|^{\frac{p+1}{p-1}} d x d t<\infty
$$

for the same $m$ and $\beta$ defined in (5.5).

Finally we can verify the inequality in condition $(i)$ using

$$
t^{-1} P \leq c w, \quad\left|P_{t}\right| \leq c w
$$

together with

$$
C \geq C^{-}, \quad t C_{t} \leq C^{-}
$$

from Lemma 5.5. The resulting lower bound is

$$
\begin{aligned}
C w-(C P)_{t} & =C w-t C_{t} \cdot t^{-1} P-C P_{t} \\
& \geq c C^{-} w .
\end{aligned}
$$

Thus conditions $(i)$ and $(v)$ hold if Lemma 5.5 holds. To complete the proof of Proposition 5.4 we will show the auxiliary result.

Proof of Lemma 5.5. The main difference with Proposition 5.3 is that $m$ can be larger than $m(V)$. Hence a negative lower bound of the form

$$
\Delta S(x)-m V(x) \geq(m(V)-m-\delta) V(x) \geq-\frac{1}{2} k_{1}(1+|x|)^{-\alpha}
$$

can not be improved. However, $\beta$ guarantees a positive bound for large $|x|$ on the difference

$$
V(x) S(x)-|\nabla S(x)|^{2} \geq c \delta V(x) S(x)-c \geq 2 k_{2}(1+|x|)^{2-2 \alpha}-c,
$$


where $k_{2}>0$. These two estimates and formula (5.3) yield

$$
\begin{aligned}
C & \geq-\frac{1}{2} k_{1} t^{-1}(1+|x|)^{-\alpha}+2 k_{2} t^{-2}(1+|x|)^{2-2 \alpha}-c t^{-2}-2 t^{-3} S(x) \\
& \geq-k_{1} t^{-1}(1+|x|)^{-\alpha}+k_{2} t^{-2}(1+|x|)^{2-2 \alpha}
\end{aligned}
$$

for sufficiently large $t \geq t_{0}$. Thus the inequality for $C$ in Lemma 5.5 is established.

The estimate of $t C_{t}$ is very similar, since $C$ is a polynomial of $t^{-1}$. There are more terms in $t C_{t}$ but its leading terms are just opposite to the corresponding terms in $C$. We can readily bound all small terms of $t C_{t}$ using $S(x) \leq c(1+|x|)^{2-\alpha}$. Such estimates will complete the proof of Lemma 5.5.

\section{Proofs of Theorem 1.3, 1.4, and Corollary 1.5}

We use the weights constructed in the previous section to derive these results from the conditional results in Proposition 4.4.

Proof of Theorem 1.3. We choose weights $w$ and $P$ defined in (5.1) with parameters $m=m(V)-2 \delta$ and $\beta=m(V)-\delta$, see (5.4). Then Proposition 4.4 yields the following weighted estimates:

$$
\begin{aligned}
\int e^{(m(V)-\delta) \frac{\phi(x)}{t}} V(x) u^{2} d x & \leq c t^{2 \delta-m(V)}, \\
\int e^{(m(V)-\delta) \frac{\phi(x)}{t}}\left(\frac{1}{t}+\frac{\phi(x)}{t^{2}}\right)^{-1}\left(u_{t}^{2}+|\nabla u|^{2}\right) d x & \leq c t^{2 \delta-m(V)}, \\
\int e^{(m(V)-\delta) \frac{\phi(x)}{t}}\left(\frac{1}{t}+\frac{\phi(x)}{t^{2}}\right)^{-1}|u|^{p+1} d x & \leq c t^{2 \delta-m(V)},
\end{aligned}
$$

where $t \geq t_{0}$ and $c$ is a constant depending on $E_{0}$ and $G_{0}$. To simplify these, we recall

$$
\phi_{0}(1+|x|)^{2-\alpha} \leq \phi(x) \leq \phi_{1}(1+|x|)^{2-\alpha} .
$$

Thus $V(x) \geq c(\phi(x))^{-\frac{\alpha}{2-\alpha}}$ with some $c>0$. We have the lower bounds

$$
V(x) \geq c t^{-\frac{\alpha}{2-\alpha}}\left(\frac{\phi(x)}{t}\right)^{-\frac{\alpha}{2-\alpha}} \geq c t^{-\frac{\alpha}{2-\alpha}} e^{-\delta \frac{\phi(x)}{t}}
$$

and

$$
\left(\frac{1}{t}+\frac{\phi(x)}{t^{2}}\right)^{-1}=t\left(1+\frac{\phi(x)}{t}\right)^{-1} \geq c t e^{-\delta \frac{\phi(x)}{t}} .
$$

The estimates in Theorem 1.3 follow with a loss of decay $2 \delta$ and large $t \geq t_{0}$. To obtain the final form we increase the constant $c_{\delta}$, if necessary, and replace $2 \delta$ by $\delta$.

Proof of Theorem 1.4. Here we use the weights $w$ and $P$ with the second set of parameters defined in (5.5). The result is again a simple consequence of Proposition 4.4 and the lower bounds on $V(x)$ and $\left(\frac{1}{t}+\frac{\phi(x)}{t^{2}}\right)^{-1}$.

Proof of Corollary 1.5. We add the three estimates in Theorem 1.3, or Theorem 1.4, and restrict the integration to $\left\{x: \phi(x) \geq t^{1+\epsilon}\right\}$ :

$$
\int_{\phi(x) \geq t^{1+\epsilon}} e^{(m(V)-\delta) \frac{\phi(x)}{t}}\left(u^{2}+u_{t}^{2}+|\nabla u|^{2}+|u|^{p+1}\right) d x \leq c_{\delta} t^{c},
$$


where $c$ depends on $\alpha, p$, and $n$. From $\phi(x) / t \geq t^{\epsilon}$ we have that

$$
\int_{\phi(x) \geq t^{1+\epsilon}}\left(u^{2}+u_{t}^{2}+|\nabla u|^{2}+|u|^{p+1}\right) d x \leq c_{\delta} t^{c} e^{-(m(V)-\delta) t^{\epsilon}} .
$$

This completes the proof, since $t^{c}$ can be included in the exponential term after a slight increase of $\delta$. Notice that the region of integration is inside the support of $u$ if $0<\epsilon<1-\alpha$.

Acknowledgments. The authors wish to express their gratitude toward Roberto Triggiani especially for his role as a catalyst in the process of writing this paper.We appreciate this very much. We also want to thank the referee for the valuable comments.

\section{REFERENCES}

[EK] Escobedo, M. and Kavian, O. Asymptotic behaviour of positive solutions of a nonlinear heat equation, Houston J. Math. 14 (1988), no. 1, 39-50.

bibitem[HKN]HKN Hayashi, N., Kaikina, E. and Naumkin, P., Damped wave equation with super critical nonlinearities, Differential Integral Equations 17 (2004), no. 5-6, 637-652.

[IM] Ikehata, R. and Matsuyama, T., Fast decay of solutions for linear wave equations with dissipation localized near infinity in an exterior domain, JDE, 188, 2003, 390-405.

[I] Ikehata, Ryo, Some remarks on the wave equation with potential type damping coefficients, Int. J. Pure Appl. Math. 21 (2005), no. 1, 19-24.

[K] Karch, G., Selfsimilar profiles in large time asymptotics of solutions to damped wave equations, Studia Math. 143 (2000), no. 2, 175-197.

[KNO] Kawashima, S., Nakao, M. and Ono, K., On the decay property of solutions to the Cauchy problem of the semilinear wave equation with a dissipative term, J. Math. Soc. Japan 47 (1995), no. 4, 617-653.

[M1] Matsumura, Akitaka, On the asymptotic behavior of solutions of semi-linear wave equations, Publ. Res. Inst. Math. Sci. 12 (1976/77), no. 1, 169-189.

[M2] Matsumura, Akitaka, Energy decay of solutions of dissipative wave equations, Proc. Japan Acad. Ser. A Math. Sci. 53 (1977), no. 7, 232-236.

[M3] Mochizuki, K., Scattering theory for wave equations with dissipative terms, Publ. Res. Inst. Math. Sci. 12 (1976/77), no. 2, 383-390.

[MN] Mochizuki, K. and Nakazawa, H., Energy decay and asymptotic behavior of solutions to the wave equations with linear dissipation, Publ. Res. Inst. Math. Sci. 32 (1996), no. 3, 401-414.

[N1] Nakao, M., Energy decay for the linear and semilinear wave equations in exterior domains with some localized dissipations, Math. Z. 238 (2001), no. 4, 781-797. 35L70

[N2] Narazaki, T., $L^{p}-L^{q}$ estimates for damped wave equations and their applications to semilinear problems, J. Math. Soc. Japan 56 (2004), no. 2, 585-626.

[NZ] Nishihara, K. and Zhao, H., Decay properties of solutions to the Cauchy problem for the damped wave equation with absorption, J. Math. Anal. Appl. 313 (2006), no. 2, 598-610.

[R] Reissig, M., $L^{p}-L^{q}$ decay estimates for wave equations with time-dependent coefficients, J. Nonlinear Math. Phys. 11 (2004), no. 4, 534-548.

[RW] Reissig, M. and Wirth, J., $L^{p}-L^{q}$ estimates for wave equations with monotone timedependent dissipation, Proceedings of the RIMS Symposium on MathematicalModels of Phenomena and Evolution Equations (to appear).

[S1S2] Shatah, J. and Struwe, M., Geometric wave equations. Courant Lecture Notes in Mathematics, 2. New York University, Courant Institute of Mathematical Sciences, New York; American Mathematical Society, Providence, RI, 1998. viii+153 pp. ISBN: 0-9658703-1-6; 0-8218-2749-9.

[S3] Strauss, W., Nonlinear wave equations. CBMS Regional Conference Series in Mathematics, 73. Published for the Conference Board of the Mathematical Sciences, Washington, DC; by the American Mathematical Society, Providence, RI, 1989. x+91 pp. ISBN: 0-8218-0725-0.

[TY1] Todorova, G. and Yordanov, B., Critical exponent for a nonlinear wave equation with damping, J. Differential Equations 174 (2001), no. 2, 464-489. 
[TY2] Todorova, G. and Yordanov, B., Weighted $L^{2}$-Estimates for Dissipative Wave Equations with Variable Coefficients, to appear.

[U] Uesaka, H., The total energy decay of solutions for the wave equation with a dissipative term, J. Math. Kyoto Univ. 20 (1980), no. 1, 57-65.

[W1] Wirth, J., Solution representations for a wave equation with weak dissipation, Math. Methods Appl. Sci. 27 (2004), no. 1, 101-124.

[W2] Wirth, J., Wave equations with time-dependent dissipation I. Non-effectivedissipation, (to appear).

Grozdena Todorova, Borislav Yordanov: University of Tennessee - Knoxville

E-mail address: todorova@math.utk.edu, yordanov@math.utk.edu 